\title{
GAUGE: the GrAnd Unification and Gravity Explorer
}

\author{
G. Amelino-Camelia - K. Aplin • M. Arndt - J. D. Barrow •
}

R. J. Bingham • C. Borde · P. Bouyer • M. Caldwell •

A. M. Cruise - T. Damour • P. D'Arrigo • H. Dittus •

W. Ertmer • B. Foulon · P. Gill • G. D. Hammond •

J. Hough • C. Jentsch · U. Johann · P. Jetzer • H. Klein •

A. Lambrecht • B. Lamine • C. Lämmerzahl •

N. Lockerbie · F. Loeffler • J. T. Mendonca • J. Mester •

W.-T. Ni • C. Pegrum - A. Peters • E. Rasel - S. Reynaud •

D. Shaul • T. J. Sumner • S. Theil • C. Torrie •

P. Touboul · C. Trenkel · S. Vitale · W. Vodel • C. Wang •

H. Ward · A. Woodgate

Received: 16 November 2007 / Accepted: 15 February 2008 / Published online: 12 June 2008

(C) Springer Science + Business Media B.V. 2008

\begin{abstract}
The GAUGE (GrAnd Unification and Gravity Explorer) mission proposes to use a drag-free spacecraft platform onto which a number of experiments are attached. They are designed to address a number of key issues at the interface between gravity and unification with the other forces of
\end{abstract}

G. Amelino-Camelia

Dipartmento di Fisica, Universita Roma "La Sapienza", Rome, Italy

K. Aplin · R. J. Bingham · M. Caldwell

Science and Technology Facilities Council, Rutherford Appleton Laboratory, Didcot, UK

M. Arndt

Institut für Experimentalphysik, Universität Wien, Vienna, Austria

J. D. Barrow

Department of Applied Mathematics and Theoretical Physics, Cambridge, UK

C. Borde

Systemes de Reference Temps Espace/Laboratoire de Physique des Lasers, Paris, France

P. Bouyer

Laboratoire Charles Fabry, Institut d'Optique, Palaiseau, France

A. M. Cruise - G. D. Hammond

University of Birmingham, Birmingham, UK 
nature. The equivalence principle is to be probed with both a high-precision test using classical macroscopic test bodies, and, to lower precision, using microscopic test bodies via cold-atom interferometry. These two equivalence principle tests will explore string-dilaton theories and the effect of spacetime fluctuations respectively. The macroscopic test bodies will also be used for intermediate-range inverse-square law and an axion-like spin-coupling search. The microscopic test bodies offer the prospect of extending the range of tests to also include short-range inverse-square law and spin-coupling measurements as well as looking for evidence of quantum decoherence due to space-time fluctuations at the Planck scale.

Keywords Fundamental physics - Space mission • Equivalence principle • Mass-spin coupling $\cdot$ Atom interferometry

T. Damour

Institut des Hautes Etudes Scientifiques, Bures-sur-Yvette, France

P. D'Arrigo · C. Trenkel

EADS Astrium, Stevenage, UK

H. Dittus · C. Lämmerzahl · S. Theil

ZARM, University of Bremen, Bremen Germany

W. Ertmer · E. Rasel

Institute for Quantum Optics, Leibniz Universität Hannover, Hanover, Germany

B. Foulon · P. Touboul

ONERA, Chatillon, France

P. Gill · H. Klein

National Physics Laboratory, Teddington, UK

J. Hough - C. Torrie · H. Ward - A. Woodgate

Institute of Gravitational Research, University of Glasgow, Glasgow, UK

C. Jentsch · U. Johann

EADS, Astrium, Friedrichshafen, Germany

P. Jetzer

Institute for Theoretical Physics, University of Zurich, Zurich, Switzerland

A. Lambrecht · B. Lamine · S. Reynaud

Laboratoire Kastler Brossel, Paris, France

N. Lockerbie $\cdot$ C. Pegrum

University of Strathclyde, Glasgow, UK

F. Loeffler

Physikalisch-Technnische Bundesanstalt, Braunschweig, Germany

Springer 


\title{
1 Introduction
}

GAUGE (GrAnd Unification and Gravity Explorer) is a proposal to the Cosmic Visions programme at ESA. The proposal is for a drag-free spacecraft platform onto which is attached a number of modular experiments. A complement of experiments is designed to address a number of key issues at the interface between gravity and unification with the other forces of nature. We include:

- A test of string-dilaton theories using a high precision macroscopic equivalence principle experiment, MEPE

- A test of the effect of quantum space-time fluctuations in a microscopic equivalence principle experiment, QSTEP

- A $1 / r^{2}$ test at intermediate ranges, IRISL

- An intermediate range axion-like mass-spin coupling search, IRSC

- Measurement of quantum decoherence from space-time fluctuations at the Planck scale, QDE

In addition there are some exciting options which need more study to confirm their feasibility and sensitivity. These are short range tests of the inverse square law (SRISL) and spin-coupling forces (SRSC) at distances of a few microns, which would provide a test of extra dimensions. MEPE will achieve an equivalence principle test between classical macroscopic test masses to 1 part in $10^{18}$. The experiment concept is simply to put test mass pairs into freefall around the Earth in what is essentially a repeat of Galileo's experiment. Four test mass pairs with three different materials are used to provide a

\author{
J. T. Mendonca \\ Instituto Superior Técnico, Lisbon, Portugal \\ J. Mester \\ Stanford University, Standford, USA \\ W.-T. Ni \\ Purple Mountain Obervatory, Nanjing, China
}

A. Peters

Humboldt University, Berlin, Germany

D. Shaul · T. J. Sumner $(\bowtie)$

Imperial College London, London, UK

e-mail: t.sumner@imperial.ac.uk

S. Vitale

University of Trento, Trento, Italy

W. Vodel

Friedrich-Schiller-Universität, Jena, Germany

C. Wang

University of Aberdeen, Aberdeen, UK 
cyclic closure condition and a null test. The technology is sound and mature. QSTEP is also an equivalence principle test but this time using atomic species, $\mathrm{Rb}$ and $\mathrm{Cs}$ and will achieve 1 part in $10^{15}$. Even though it is less sensitive than MEPE it will allow a meaningful test within a quantum space-time fluctuation which MEPE could not. ISL will allow searches for inverse square law deviations at two scale lengths; intermediate using classical proof mass technology and short-range using atom interferometry. SC will allow searches for spin-mass coupling at two scale lengths; intermediate using classical proof mass technology and short-range using atom interferometry. QDE will use atom interferometry to look for fundamental limitations to the quality of matter wave interference patterns due to space-time fluctuations. MEPE and some parts of IRISL and IRSC rely on superconducting technology and hence require low temperature operation inside a liquid helium dewar. QSTEP, SRISL, SRSC and QDE require the use of atom interferometry and this is a room temperature technology. The cold experiments will be accommodated inside a liquid helium dewar which is attached to the spacecraft. The warm experiments will fit inside the spacecraft bus itself. The payload can be accommodated on a replica of the LISA Pathfinder bus. The bus should also include the LTP sensors to provide drag-free capability at the bus level as well as take part in some of the experiments themselves. The whole spacecraft is then compatible with a launch on VEGA into a sun-synchronous orbit. The experiments will be carried out in sequence starting with those dependent on the helium supply. The overall mission duration will be 2 years.

\section{Scientific objectives}

GAUGE proposes new tests of general relativity (GR) in Earth-orbit that will include the Einstein equivalence principle (EEP), the inverse square law, spincoupling and the quantum vacuum. This will be done using a combination of macroscopic test masses and matter wave interferometry in a drag-free spacecraft.

\subsection{The equivalence principle}

The EEP is the basis of Einstein's theory of GR and is one of the most enduring invariants of nature. There are good reasons based on current theoretical models looking for a unified quantum theory of matter and fields that this invariance will break down at levels below the present limits. In these models GR, though very nearly true, describes a field structure that evolved from a more complex unified field structure in the early universe that violated the equivalence principle. GAUGE will therefore have a direct bearing on the "Structure and Evolution of the Universe", as well as probing the standard model (SM) of particle physics. The SM predicts a grand unification of the forces of nature at the grand unification theory (GUT) scale. There is experimental evidence for unification of the electromagnetic and weak forces, but the 


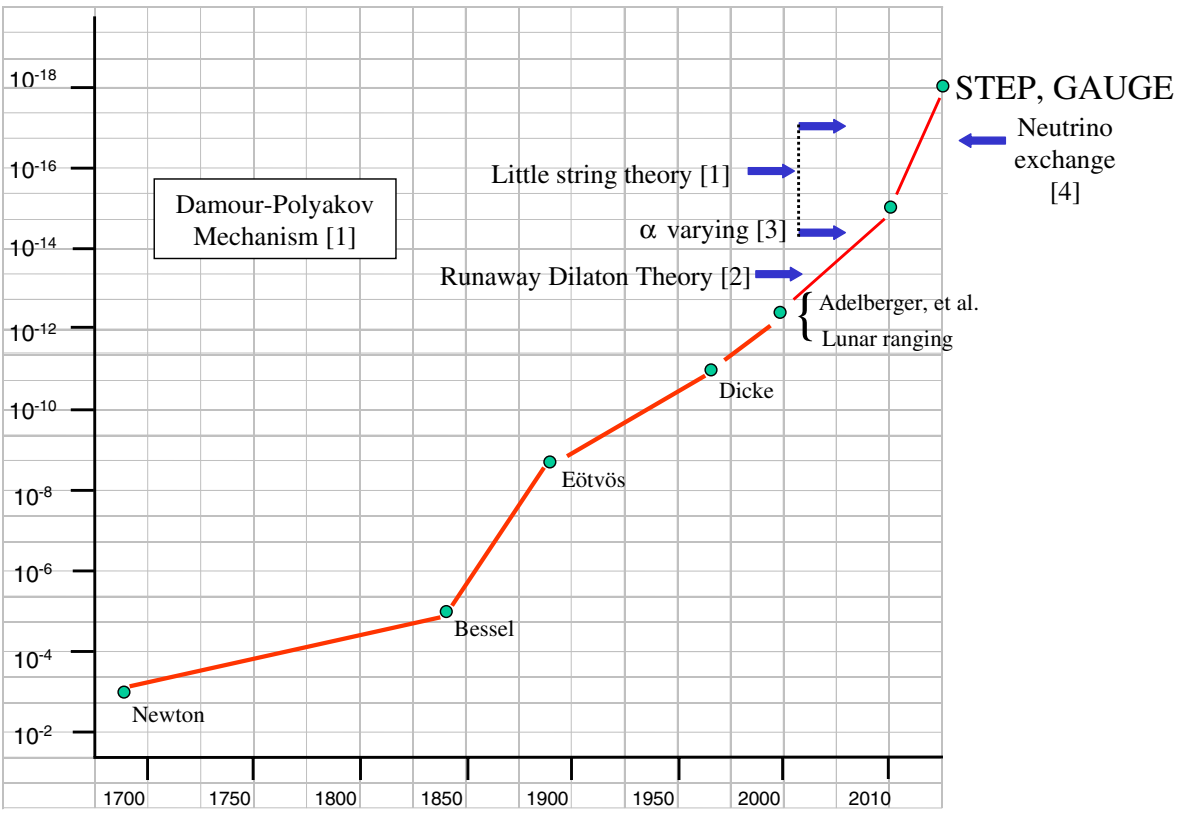

Fig. 1 Progression in sensitivity of EP measurements

resulting electro-weak force still remains distinct from the strong force but with a trend to unify at the GUT scale. These forces remain totally disconnected from the force of gravity. Another problem arising with the standard model is the "gauge hierarchy problem". Electroweak unification occurs through a matching of forces at one mass scale, but the mass scale for grand unification differs from this by a factor $10^{12}$ well beyond test using present and future particle accelerators. The pursuit of unification through string theory and supersymmetry has resulted in the need to involve extra dimensions and naturally energizing new long range forces with strength comparable to or weaker than gravity. These forces couple not to mass, but to other properties of the atom like baryon and lepton number in various combinations, and they may violate the equivalence principle at a level that is measurable by GAUGE (see [1,2] and Fig. 1). Also shown is the predicted level at which cosmological models incorporating a time varying alpha invoke violations [3] and the level at which an anomalous coupling to the weak interaction could appear due to neutrino-antineutrino exchange [4]. An EEP violation would confirm the existence of new forces. A confirmation to one part in $10^{18}$ would extend GR theory and restrict the existence of new ultra-weak long-range forces causing a major problem for "string theory".

\subsection{Inverse square law}

The inverse square law can be checked with both classical and quantum detectors on GAUGE. GAUGE will use pairs of masses to measure force 
changes as one of the masses is moved. The inverse square law is well-tested for planetary astrophysical distances but not for distances of microns or less. Models that go beyond the SM such as string theory and supersymmetry aiming at unification have, as a natural consequence, new long range forces with strengths comparable or weaker than gravity. GAUGE will be able to look for violations of Newton's law confirming new long range forces on both intermediate $(\sim 20 \mathrm{~cm})$ and possible short range $(\sim 10 \mu \mathrm{m})$.

\subsection{Spin coupling}

In addition to new long range forces modern unification theories also predict the existence of new light-mass pseudoscalars. These give rise to a force between intrinsic quantum mechanical spin and mass. So far all attempts to detect spin-coupling forces either cosmologically or in the laboratory have produced only upper limits. Hypotheses about spin-mass coupling forces originate in attempts to resolve the problems of the standard model such as the charge parity conservation puzzle and the composition of dark matter. The existence of spin-mass coupling also implies a violation of the EEP. The SM is unquestionably a highly successful description of the electromagnetic, weak and strong interactions comprising the gauge symmetry groups $U(1) \times S U(2) \times S U(3)$. Although there is no current disagreement between experiment and theory the SM is known to have some shortcomings. Perhaps one of the most puzzling is the fact that the QCD Lagrangian contains a term that can violate $\mathrm{CP}$ symmetry

$$
L=\theta \frac{\alpha}{8 \pi} G^{\mu v} G_{\mu v}
$$

where $\theta$ is an arbitrary parameter and $G^{\mu v} / G_{\mu v}$ are the gluon field tensors. Experimental limits on the electric dipole moment of the neutron suggest that this term is suppressed by 10 orders of magnitude $\left(\theta<2.5 \times 10^{-10}\right)$ and this is the well-known strong CP problem. An elegant solution to this problem is the addition of a additional $U(1)$ symmetry called the Peccei-Quinn group [5]. This symmetry is broken at an energy scale $f_{a}$ and gives rise a spin-zero boson named the axion, whose field, $\Phi_{a}$, couples to the gluon field via an additional term in the QCD Lagrangian

$$
L=\left(\theta-\frac{\Phi_{a}}{f_{a}}\right) \frac{\alpha}{8 \pi} G^{\mu v} G_{\mu v}
$$

The axion potential is minimised when $\Phi_{a}=\theta \times f_{a}$ and the CP violating term is driven to zero. Although the axion is the best motivated spin-zero boson, in principle many axion-like particles could exist in nature. In general field theories that spontaneously break global symmetry result in a massless spinzero Goldstone boson. If the symmetry is not exact the boson can acquire a small mass (as in the case of the axion). Other examples are the U-boson [6], the arion [7], the familon [8] and the dilaton [9]. Such particles have small masses and couple very weakly to normal matter, making them potential nonbaryonic dark matter candidates. 
Axions, and axion-like particles, couple to both the scalar (or spin independent) and pseudoscalar (spin dependant) vertex [10]. This causes three distinct interactions: scalar-scalar (mass-mass), scalar-pseudoscalar (massspin) and pseudoscalar-pseudoscalar (spin-spin). An interaction of the massmass type would lead to a violation of the inverse square law of gravity in the range $20 \mu \mathrm{m}<\lambda<2 \mathrm{~cm}$. However this is difficult to demonstrate in a "clean measurement" for axions. The spin-spin interaction is also difficult because the direct magnetic coupling between the aligned fermions (or spins) has to be shielded. As a result the most commonly measured interaction is the massspin. The interaction potential between a polarised spin $\sigma$ and a nucleon is

$$
V=g_{p} g_{s} \frac{\hbar^{2}}{8 \pi m_{e}} \hat{\sigma} \cdot \hat{r}\left(\frac{1}{\lambda r}+\frac{1}{r^{2}}\right) \exp (-r / \lambda)
$$

where $g_{p} g_{s}$ is a dimensionless coupling constant, $m_{e}$ is the mass of the electron (assuming the aligned spins are polarised electrons) and $\lambda$ is the interaction range. This interaction violates parity and time reversal symmetries. Its detection would be a first in nature. The axion is described by two parameters, $f_{a}$ and $\theta$ to which the mass, range and coupling constants are related by:

$$
\begin{array}{r}
m_{a} \simeq 10^{-5} \mathrm{eV}\left(10^{12} \mathrm{GeV} / f_{a}\right) \\
\lambda \simeq 2 \mathrm{~cm}\left(f_{a} / 10^{12} \mathrm{GeV}\right) \\
g_{p} g_{s}=\frac{\theta}{\lambda^{2}} \times 6 \times 10^{-33}
\end{array}
$$

Astrophysical arguments constrain the mass and energy of the axion. Axions produced in stars and supernovae can escape easily and provide a significant cooling mechanism. The neutrino burst duration from SN1987A implied $m_{a} \leq$ $10 \mathrm{meV}$ and $\lambda \geq 20 \mu \mathrm{m}$ [11]. Conversely $m_{a} \geq 1 \mu \mathrm{eV}$ and $\lambda \leq 20 \mathrm{~cm}$ come from a cosmological need to avoid their over-production in the early universe. Experimental searches probe axions either through their coupling to photons ( 2 photon-axion vertex) or though the mass-spin interaction of (3). The former are typically resonant cavity searches such as ADMX [12], the CernAxion-Solar-Telescope (CAST) [13] or the PVLAS [14] search for vacuum birefringence. The latter search for axions through their mass-spin (or spinspin) interactions. At ranges $>1 \mathrm{~m}$, a torsion balance with an attached spinpolarised test-mass [15] sets the tightest constraints. In the range $10 \mathrm{~cm}<\lambda<$ $1 \mathrm{~m}$ the best constraints come from comparison of the relative precession frequencies of $\mathrm{Hg}$ and $\mathrm{Cs}$ magnetometers [16]. Several other experiments have placed limits at considerably shorter ranges. A DC SQUID magnetometer used to measure the change in polarisation of a paramagnetic salt induced by the motion of an unpolarised source mass [17] sets the strongest constraints for $5 \mathrm{~mm}<\lambda<10 \mathrm{~cm}$. The best limits at ranges $<5 \mathrm{~mm}$ come from a spherical torsion balance operating at $4.2 \mathrm{~K}$ [18]. In this experiment unpolarised test masses located on the torsion balance are subjected to the possible axion field produced by a polarisable source of spins of the type proposed for GAUGE. The dimensionless coupling constant at a range of $1 \mathrm{~mm}$ was reported to be $g_{p} g_{s}<1.0 \times 10^{-24}$. 


\subsection{Quantum vacuum}

\subsubsection{Violation of equivalence principle at the atomic level}

A number of models describe space-time fluctuations as small fluctuations of the metric on a given background metric. Even though the unification of quantum mechanics and gravitation is one of the big outstanding problems of contemporary physics there is no theory of quantum gravity. However, approaches like string theory or loop quantum gravity make some general predictions which may serve as guidance for experimental search. The difficulty is that in many cases the precise strength of the various expected effects are not known. Quantum gravity phenomenology tries to overcome this problem by parameterizing possible effects and working out experimental consequences for classes of phenomena. Different quantum gravity theories are characterized by different parameters. One class of expected effects of 'quantum gravity' is a non-trivial quantum-gravity vacuum ("space-time foam") which can be regarded as a fluctuating space-time. Space-time fluctuations could lead to a minimal observable distance setting an absolute bound on the measurability of distances and defining a fundamental length scale [19, 20]. For instance the search for additional noise sources in gravitational wave interferometers was previously considered, and analyzed in the context of an experiments with optical cavities and resonators [21, 22]. A space-time foam may also violate the principle of equivalence as suggested by Ellis and coworkers [23]. Another prediction of quantum gravity models are so called deformed dispersion relations [24-27]. Hu and Verdauger [28] analyzed classical stochastic fluctuations of space-time geometry stemming from quantum fluctuations of matter fields in the context of semiclassical theory of gravity. This leads to a stochastic behaviour of the metric tensor. Effects of fluctuations of space-time geometry leading to, e.g., lightcone fluctuations, redshift and angular blurring were discussed in [30]. The analysis in [16] takes into account non-conformal fluctuations of the metric and yields a modified inertial mass subject to the stochastic properties. The dominant modification is through an anomalous inertial mass tensor which depends on particle type and fluctuation scenario. This necessarily violates the weak equivalence principle (WEP) and, in general, also Lorentz invariance. By means of the rescaled inertial mass and the dependency on particle type $\left(\Delta t_{p}\right)$ one can guess that the WEP may be violated with a ratio of inertial to gravitational mass given by

$$
\left(\frac{m_{g}}{m_{i}}\right)_{p}=1+\alpha\left(\gamma, \Delta t_{p}\right) \text { with } \alpha\left(\gamma, \Delta t_{p}\right)=\Sigma_{j=1}^{3}\left\langle\tilde{h}^{i j}\right\rangle
$$

where the index $p$ stands for "particle". $\alpha$ is proportional to the particle resolution time $\Delta t_{p}^{-1}$ and therefore, identifying the resolution scale with the Compton time, $t_{C}$, is subject to an amplification when large coherent states are chosen scaling with the number of particles. The resolution time for Caesium is $\Delta t_{p j} \simeq 10^{-26}$ and for Hydrogen $\Delta t_{p 2} \simeq 10^{-24}$. As the variance is given by the 
second order perturbation term $\tilde{h}$ one can infer that for linear perturbations of about $h \simeq 10^{-20}$ we obtain (leaving rotational invariance untouched $\alpha_{j 1}=\alpha$ ).

$$
\alpha_{1}=\frac{10^{-40}}{10^{-26}}=10^{-14} \text { Caesium } \alpha_{2}=\frac{10^{-40}}{10^{-24}}=10^{-16} \text { hydrogen }
$$

The Eötvös factor characterising the WEP violation then reads

$$
\eta=2 \frac{\left|a_{1}-a_{2}\right|}{\left|a_{1}+a_{2}\right|}=\frac{\left|\alpha_{1}-\alpha_{2}\right|}{1+\frac{\alpha_{1}-\alpha_{2}}{2}}
$$

Since fluctuations influence only the dynamics of quantum particles, it can best be tested with atomic interferometry. Space missions like GAUGE [25] may provide improved sensitivities. Here we took space-time fluctuations as classical fluctuations of the metric. The effect can be amplified significantly if large coherent systems (e.g. BECs) are taken into account, scaling with the number of particles, or by choosing particle species with small time resolution scales. Generally, our results concerning the amplitude of the effects as a function of resolution scale are also viable for the case where rotational invariance is still valid $\left(\tilde{\alpha}^{11}=\tilde{\alpha}^{22}=\tilde{\alpha}^{33}\right)$. Thus our model incorporates several effects which are possible outcomes of a theory of quantum gravity.

\subsubsection{Quantum vacuum atom wave decoherence}

The physics of the vacuum is amongst the most fundamental issues of quantum field theory and GR. The existence of zero point energy is an essential feature of quantum field theory [31]. EM vacuum energy has already seen experimental confirmation through the Casimir effect [32], Lamb shift [33] and spontaneous emission [34]. Recent measurement of the Planckian spectral density of Josephson noise in superconducting circuits [35] confirms the physical reality of EM zero point energy and its ability to interact with the macroscopic world. According to the uncertainty principle, all field components in the SM (and possibly beyond) show a minimum level of fluctuation even at absolute zero temperature, giving rise to zero point energy in vacuum. The role of vacuum energy in cosmology is a subject of intense debate [31, 36, 37]. Does vacuum energy gravitate? The Planckian spectrum for zero point energy is Lorentz invariant but has no UV cutoff, resulting in a formally divergent energy density. The only natural UV cutoff frequency seems to be the Planck frequency but if this value is used the resulting finite vacuum energy density would provide a 'cosmological constant' $10^{120}$ times above the observational limit, measured to be a fraction of the critical density of the Universe. This is the well known cosmological constant problem [31, 38, 39]. A UV cutoff imposed on the Planckian spectrum will lead to Lorentz invariance. Could a signature of this be detectable [29]? The lack of experimental guidance on the gravitational nature of vacuum energy and its role in cosmology has led to many theoretical speculations including quintessence, braneworld and holographic approaches [31,38]. Quantum field theory in curved spacetime and semiclassical gravity are useful theoretical tools in estimating gravitational effects on quantum fields and their back-reactions [40]. It has important 
applications in, e.g. black hole radiation and evaporation [41]. However, semiclassical gravity has been shown to contradict certain experiments [42]. Theoretically, it fails to capture quantum fluctuations of spacetime and would lead to a perfectly homogeneous and isotropic universe even in the presence of fluctuating quantum matter fields [43]. Stochastic gravity [28] is probably a better approximation to quantum gravity but so far has been subject to few experimental tests. The ubiquity of vacuum energy makes all quantum systems into open systems [44]. For example, EM zero point energy may affect electron diffusion in metals [45]. Furthermore, decoherence of free electrons in vacuum by EM zero point energy has been a subject of intense investigations [46, 47]. Due to the weakness of gravitational coupling, the gravitational analogue of the Casimir effect is inconceivable. However, quantum decoherence due to gravitational vacuum fluctuations does not require modifying the boundary conditions of gravity and could be tested using matter wave interferometry under ultra quiet conditions. A number of models exploring quantum and stochastic gravitational decoherence have recently been considered [48-51]. These phenomenological models predict decoherence in terms of the loss of visibility in matter wave interferometry of the form

$$
\Delta V \simeq-A m^{2} t
$$

where $m$ is the mass of the quantum particle (which may be an atom like Cs or molecule such as $\mathrm{C}_{70}$ ), $t$ is the flight time and $A$ is a model dependent form factor. It depends on whether the decoherence effect is due to, e.g. Newtonian gravitational interaction $[48,51]$ or stochastic gravitational waves [50] or zeropoint gravitational fluctuations [51] which also takes into account nonlinear gravitational effects. Furthermore, for cooled and hence indistinguishable quantum particles the form factor $A$ is expected to increase quadratically with the particle number $N$. In the approach of [51], this factor depends on a UV cutoff frequency $w_{p} / l$ with a phenomenological parameter $\lambda$ given by:

$$
A \simeq \frac{N^{2}}{l^{3} m_{P}^{2} t_{P}}
$$

where the subscript $P$ denotes a Planck scale quantity, such that $t_{P}$ is the Planck time. Theoretically, the parameter $l$ may range from order 1 (Planck cutoff scale) to $l \sim m_{P} / m$ (Compton cutoff scale). Ground based experiments place a lower limit on $l$ as $l>10^{4}$. An experimental increase in the limit will provide guidance on effective quantum gravity energy scales, especially in relation to (non)renormalization properties of quantum gravity. In order to test (10) and determine the model dependent form factor $A$ in relation to (11), GAUGE atom interferometry experiments will be carried out by varying the particle measurement parameters including flight time $t$, particle mass (using two species: $\mathrm{Cs}$ and $\mathrm{Rb}$ ) as well as particle numbers $N$. Environmental parameters including pressure and temperature will be monitored to determine the possible level of gravitational decoherence effects.

This section has discussed a range of experiments targeting a number of different scientific goals. The main underlying theme has been at the interface 
between gravity and quantum mechanics. Any discovery in this regime could lead to fundamental new insights into quantum-gravity or other new forces of nature [52] and would be of profound importance.

\section{Proposed payload instrument complement}

\subsection{Overview of all payload elements}

The scientific payload comprises the following payload elements:

- Macroscopic test-masses (LTP) used as source masses for an inverse square law test (IRISL) and as measurement masses for a spin-coupling measurement (IRSC)

- An atom interferometer used for both a microscopic equivalence principle test (QSTEP) and a quantum decoherence study (QDE)

- Three macroscopic test mass pairs housed within a cryogenic science module (CSM) used for a high-precision equivalence principle test (MEPE)

- A polarisable spin-source within the CSM to drive spin-mass coupling forces to be measured by both the LTP test masses and the test mass pairs within the CSM

- An option to supplement the atom interferometry with atom lasers which would enable both short-range inverse square law and spin-coupling tests (SRISL, SRSC)

The payload elements and their layout are identified in Fig. 2.

\subsection{The LISA test package}

The LISA Test Package (LTP) should be replicated in full. The only change required for GAUGE is to re-orientate the optical bench along the spacecraft axis. In all other respects it should have the same performance parameters as it does for LISA Pathfinder.

\subsection{Atom interferometry, QSTEP}

The high precision atom interferometer (QSTEP) payload will test the universality of free fall, using the Earth as the source mass with a differential measurement between two species of laser-cooled atoms having different nuclear properties and masses. $\mathrm{Rb}$ and $\mathrm{Cs}$ are the baseline. Options are ${ }^{87} \mathrm{Rb}$ and ${ }^{85} \mathrm{Rb}$, in bosonic atomic ensembles, and $\mathrm{Rb}$ and ${ }^{40} \mathrm{~K}$ or ${ }^{41} \mathrm{~K}$ where ${ }^{40} \mathrm{~K}$ is a fermion. Atom interferometers in the Mach-Zehnder configuration, (left in Fig. 3) are highly sensitive to inertial forces. In the time domain configuration (right in Fig. 3) proposed for GAUGE each individual atom in the ensemble is coherently split, re-directed and re-combined in a well defined time sequence. The coherent partial wave packet manipulation relies on applying coherent pulses of light which couple the two atomic ground states. This is done with two counter-propagating 'Raman' laser beams. An atom absorbs a photon 

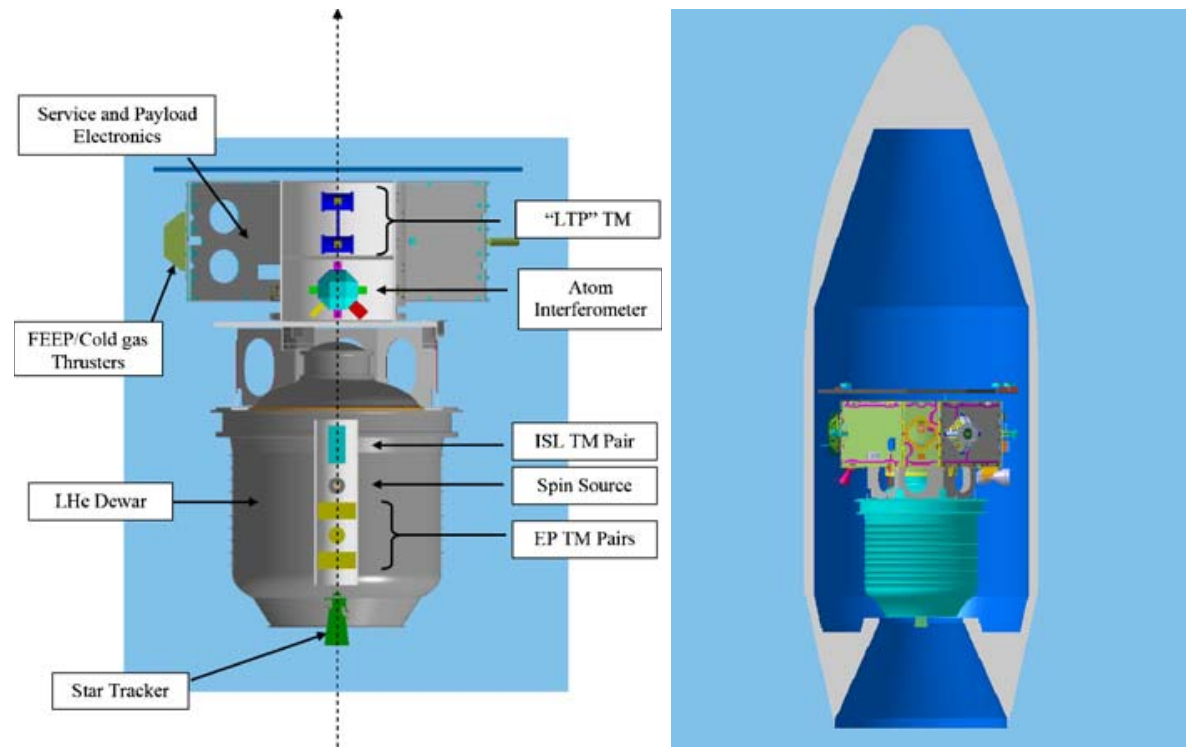

Fig. 2 GAUGE payload element identification and launch configuration

out of one laser beam and is stimulated by the other to re-emit a photon. The recoil momenta of the photons are coherently transferred to the atomic wave, such that it is either equally split, deflected or re-combined. The axis of the photon momentum (laser beam) defines the sensitive direction, and only accelerations in this direction can be sensed. The detection after the third Raman interaction measures the fraction of atoms in each of the two ground states. The ratio between the two ground states gives the phase shift and allows the acceleration to be determined. The two atomic species will be interrogated simultaneously and the difference between them contains the equivalence principle measurement.

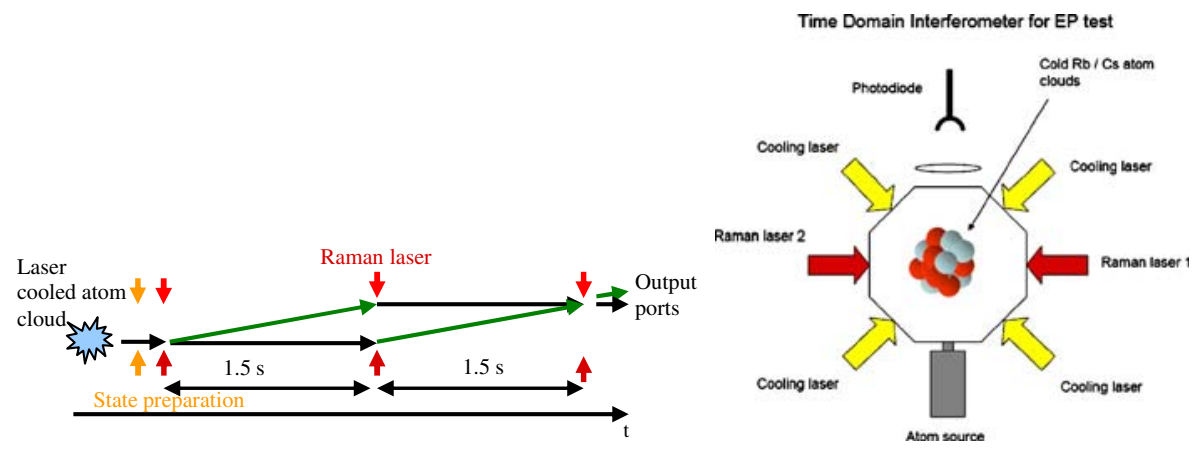

Fig. 3 High precision cold atom interferometry in Mach-Zehnder and time domain configurations 
For GAUGE the sensitive axis of QSTEP must be perpendicular to the symmetry axis of the satellite which points in sun direction. Hence, during one orbit the measurement axis will twice align with the Earth direction and twice with the tangent to the orbit path. An equivalence principle violation signal will be modulated once per orbit whereas a modulation due to the gravity gradient will be twice per orbit.

Several payload components are required for the QSTEP:

- In the central vacuum chamber where the atoms are probed the required vacuum level of order $10^{-9}$ mbar will be maintained by a small ion getter pump and additional getter materials. All lasers are fibre coupled to the beam expander telescopes which are close connected to the chamber. The detection unit contains calibrated photodiodes and a CCD camera for spatially resolved detection. The whole device will be thermally de-coupled from the LPSM. Thermal stability of the physics package is achieved by a combination of passive isolation (multi-layer insulation, conductive de-coupling with titanium mounts), and active temperature control by heaters. Extensive use of CFRP gives superior stiffness and minimises mass and thermal distortions. This vacuum chamber has to be placed in the central tube of the LPSM right below the LTP test mass housing as shown in Fig. 2.

- The lasers and modulation units required to cool, prepare and detect the atoms can be placed in one of the free outer compartments of the LPSM. The light is delivered to the central vacuum chamber using optical fibres.

- Raman laser systems will generate the laser light pulses. Two pre-stabilised lasers will be phase-locked to highly stable microwave frequency reference sources. These units can be placed outside of the inner cylinder with fibreoptic feeds.

- Computer control will autonomously run measurements, calibrations and control phases. In addition, a pre-processing of raw data will be performed. This control unit will be combined with the LPSM on-board computer.

The free fall of the two atom species should be compared with an accuracy of 1 part in $10^{15}$. If the interferometer noise is determined by shot noise the sensitivity to accelerations $\Delta a$ per shot is $\Delta a=N^{-1 / 2}\left(k_{\text {eff }} T^{2}\right)^{-1}$ where $N$ is the total number of detected atoms, $T$ is the time between the Raman laser pulses and $k_{\text {eff }}$ the effective wave vector characterising the momentum transfer. As this is inversely proportional to $T$ long interrogation times are preferred which is one of the reasons for carrying out these experiments in space. However, the residual temperature of laser-cooled atoms is a few $\mu \mathrm{K}$ corresponding to an expansion of the cloud diameter at $3 \mathrm{~cm} / \mathrm{s}$ which limits $T$ to a few seconds. Taking $T=1.5 \mathrm{~s}$ gives a total interferometer duration of $3 \mathrm{~s}$. With $N=10^{8}$ atoms the sensitivity per interferometer cycle becomes $2.76 \times$ $10^{-12} \mathrm{~m} / \mathrm{s}^{2}\left(k_{\mathrm{eff}}=22 \pi / \lambda_{\mathrm{Rb}}, \lambda_{\mathrm{Rb}}=780 \mathrm{~nm}\right)$. Assuming well behaved noise this integrates down to $\simeq 2.4 \times 10^{-15} \mathrm{~m} / \mathrm{s}^{2}$ in $1.3 \times 10^{6}$ cycles corresponding to $4 \times 10^{6} \mathrm{~s}$ or about 50 days. 


\subsection{Inverse square-law experiments}

The proposed GAUGE intermediate range IRISL test is based on the use of the two LTP test masses, in conjunction with the IRISL test mass in the cryogenic module. These three masses are arranged in-line, and each of them can be used as a sensor as well as a source, and also as a drag-free reference sensor. The experimental principle is to measure the ratio between force changes experienced by masses $m_{1}$ and $m_{2}$ when $m_{3}$ is moved, and to verify that this ratio is as expected given the ratio of the separations $r_{12}$ and $r_{13}$ according to the ISL, $\left(\frac{\Delta F_{N}\left(r_{13}\right)}{\Delta F_{N}\left(r_{23}\right)}\right)=\frac{m_{1}}{m_{2}}\left(\frac{r_{23}}{r_{13}}\right)^{3}$ down to the resolution limit of the experiment. The ratio will be estimated from $\frac{\Delta F_{N}\left(r_{13}\right)}{\Delta F_{N}\left(r_{23}\right)}=\frac{x_{1} k_{1}}{x_{2} k_{2}}$ where $x_{i}$ denotes the observed displacement of mass $m_{i}$, and $k_{i}$ the stiffness of its respective sensor. Assuming that $x_{i}$ can be measured to sufficient accuracy a meaningful verification of the ISL therefore depends on determining, to sufficiently high accuracy, the ratio between sensor stiffnesses, and the ratio between separations. One other assumption, in what follows, is that the ratio between mass values is known to better than $1 \mathrm{ppm}$. In the precision measurements of the IRISL, the following systematic effects can limit the experimental sensitivity:

- Metrology, in particular the absolute separation between the centres of mass

- Absolute calibration of force sensors (if non-null experiment)

- Density inhomogeneities present in the interacting bodies

For GAUGE, it is proposed to carry out a precision test of the ISL of gravitation at ranges between $10 \mathrm{~cm}$ and $1 \mathrm{~m}$, minimising the effect of systematics by exploiting:

- The presence of an external, well-known modulated Earth gravity gradient that can be used for absolute calibration of separation ratios

- The flexibility to use free-floating test masses alternatively as source and test masses, which can be used to calibrate stiffness ratios of force sensors

- A high force sensitivity, masses that are separated by distances much larger than their dimensions can be used, thus minimising density inhomogeneity problems

A 'proof mass' acceleration will be measured when the position of "source" masses between $30 \mathrm{~cm}$, and $1 \mathrm{~m}$ away are modulated periodically. For simplicity $2 \mathrm{~kg}$ masses will be assumed throughout (including that in the cryogenic IRISL). Modulation amplitudes of $d=4 \times 10^{-3} \mathrm{~m}$, compatible with the LTP IS gaps, give acceleration signals between $10^{-12}$ and $4 \times 10^{-11} \mathrm{~ms}^{-2}$. An LTPlike electrostatic sensor acceleration noise of $3 \times 10^{-14} \mathrm{~ms}^{-2} / \sqrt{\mathrm{Hz}}$ at $1 \mathrm{mHz}$ will be assumed for all sensors [53]. Then in $10^{6} \mathrm{~s}$ we can resolve the induced accelerations to better than $1 \mathrm{ppm}$ at $30 \mathrm{~cm}$ and $30 \mathrm{ppm}$ at $1 \mathrm{~m}$. Along the IRISL sensitive (axial) axis, use of the LTP laser interferometry can mitigate noise coupled from the spacecraft, leaving a residual acceleration noise of $7 \times 10^{-15} \mathrm{~ms}^{-2} / \sqrt{\mathrm{Hz}}$, improving the resolution at $1 \mathrm{~m}$ to about $7 \mathrm{ppm}$. There 
is the option of measuring the relative axial spacecraft to TM motion using the laser. The coupling spacecraft-TM can be calibrated by "shaking" the spacecraft deliberately and measuring the TM response. The TM noise can then be corrected for this effect. The gravitational force between two masses separated by $r_{13}$, including an additional composition-independent Yukawa potential can be parameterised as

$$
F_{N+Y}\left(r_{13}\right)=G \frac{m_{1} m_{3}}{r_{13}^{2}}\left(1+\alpha\left(1+\frac{r_{13}}{\lambda}\right) \exp \left(-r_{13} / \lambda\right)\right)
$$

where $\alpha$ characterises the strength of the interaction (relative to gravity) and $\lambda$ its range. Modulating the distance of mass $m_{3}$ by $d_{3}$ causes a modulated force on $m_{1}$

$$
\Delta F_{N+Y}\left(r_{13}\right)=-2 G \frac{m_{1} m_{3}}{r_{13}^{3}}\left(1+\alpha\left(1+\frac{r_{13}}{\lambda}+\frac{1}{2}\left(\frac{r_{13}}{\lambda}\right)^{2}\right) \exp \left(-r_{13} / \lambda\right)\right) d_{3}
$$

A second mass $m_{2}$ at a different distance $d_{23}$ from $m_{3}$ will see a different force and the ratio between the two will be

$$
\left(\frac{\Delta F_{N+Y}\left(r_{13}\right)}{\Delta F_{N+Y}\left(r_{23}\right)}\right)=\frac{m_{1}}{m_{2}}\left(\frac{r_{23}}{r_{13}}\right)^{3} \frac{1+\alpha\left(1+\frac{r_{13}}{\lambda}+\frac{1}{2}\left(\frac{r_{13}}{\lambda}\right)^{2}\right) \exp \left(-r_{13} / \lambda\right)}{1+\alpha\left(1+\frac{r_{23}}{\lambda}+\frac{1}{2}\left(\frac{r_{23}}{\lambda}\right)^{2}\right) \exp \left(-r_{23} / \lambda\right)}
$$

and Yukawa terms will escape detection if

$$
\left(\frac{\Delta F_{N+Y}\left(r_{13}\right)}{\Delta F_{N+Y}\left(r_{23}\right)}\right)-\frac{m_{1}}{m_{2}}\left(\frac{r_{23}}{r_{13}}\right)^{3} \leq \frac{m_{1}}{m_{2}} 3\left(\frac{r_{23}}{r_{13}}\right)^{3} \delta\left(\frac{r_{23}}{r_{13}}\right)\left(\frac{r_{13}}{r_{23}}\right) / \frac{r_{13}}{r_{23}}
$$

Assuming $r_{13}=1.0 \mathrm{~m}, r_{23}=0.4 \mathrm{~m}$ and $\delta\left(r_{13} / r_{23}\right) /\left(r_{13} / r_{23}\right)=2 \times 10^{-6}$ a null result from IRISL would result in limits improving on existing limits for ranges between $10 \mathrm{~cm}$ and $3 \mathrm{~m}$ by up to two orders of magnitude. A shortrange test of the ISL, SRISL, is also possible in principle using atom interferometry. A mass close to one of the matter wave paths in a spatially dispersed arrangement (Fig. 4) gives an additional phase shift of $\delta \phi=$ $\frac{1}{\hbar} \int \frac{d r}{v} V(r)$, with $V(r)=-\frac{G M m}{r}[1+\alpha \exp -r / \lambda]$. Assuming $10^{8}$ caesium atoms
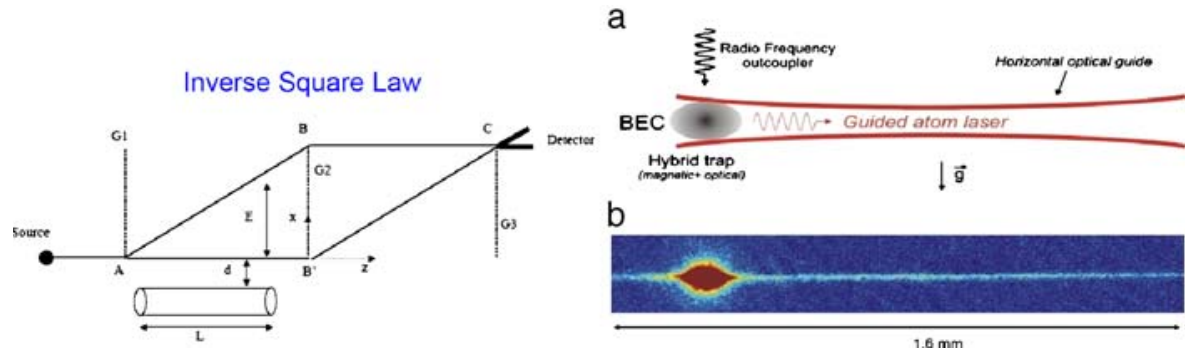

Fig. 4 A short-range ISL test using atom interferometry. Left is a general schematic. Right a Principle of an atom laser guided in an optical tweezer. b Image of the laser after $50 \mathrm{~ms}$ of propagation in the guide using $\mathrm{Rb}$ atoms 

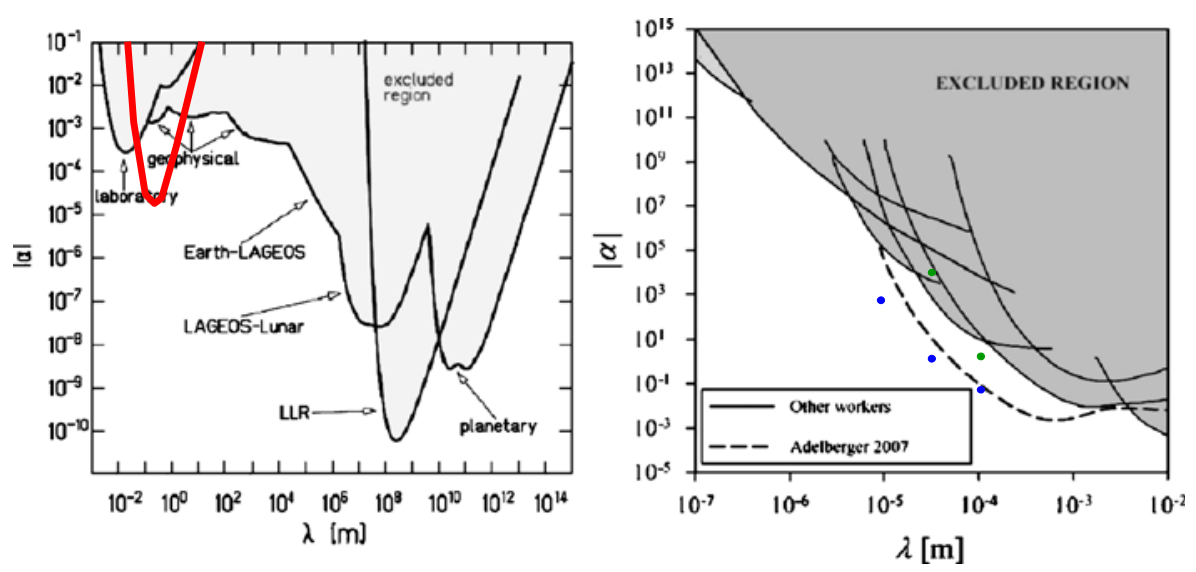

Fig. 5 Sensitivity of GAUGE ISL test to composition-independent Yukawa forces; left is for IRISL, right is for a single-shot SRISL with an over-simplified analysis

traveling at $1 \mathrm{~m} / \mathrm{s}$ with an effective temperature $\sim 1 \mu \mathrm{K}$, and a $25 \mathrm{~cm}$ long gold source mass. The right-hand panel in Fig. 5 shows the sensitivity (blue and green dots using different integration techniques) of an idealised single-shot measurement compared with current ground-based results. In principle the sensitivity should improve as $\sqrt{N}$ where $N$ is the number of repeat shots which could be $10^{6}$. However a major limitation is beam divergence which is much larger than the $75-375 \mu \mathrm{m}$ separations assumed in the simplistic calculations so far. This option is worthy of further study but is not yet on firm enough ground to adopt as baseline. One improvement might be to use a guided atom laser source [54]. This was realized starting from the combination of a magnetic trap and a horizontal optical guide, created by a laser Nd:YAG to $1,064 \mathrm{~nm}$ with a waist of $20 \mu \mathrm{m}$ (see Fig. 4). Optical and magnetic potentials create a very confined hybrid trap in which the atoms can be condensed. A low-amplitude radio frequency field transfers a part of the atoms from the trapped state $\mid F=$ $1, m_{f}=-1>$ towards the untrapped state $\mid F=1, m_{f}=0>$, and a coherent matter wave propagates in the potential formed by the optical guide. A major quality of this device is that the position and direction are controlled by the laser stability and it is possible to achieve better than $1 \mu \mathrm{m}$ RMS and $.1 \mu \mathrm{rad}$ RMS respectively. The devices can be integrated on minitraps or atomic chip, thus lowering power consumption and volume (mass). Also shown in Fig. 5 (left-hand panel) is the projected sensitivity for the IRISL experiment.

\subsection{Spin-coupling experiments}

The spin-coupling experiments search for forces between unpolarised nucleons and a spin polarised body (called the spin-source). IRSC proposes to achieve a sensitivity of $g_{p} g_{s}=6 \times 10^{-32}$ at a range of $10 \mathrm{~cm}$ improving on previous work by approximately 3 orders of magnitude. A schematic of IRSC is shown in Fig. 6 where unpolarised nucleons constitute one of the superconducting 
Fig. 6 a Schematic view of the IRSC showing the unpolarised test mass and the spin-source together with the shielded end caps, b Top view of the experiment showing the location of the Hall probe (HP)/SQUIDs and heaters (H)/thermometers (T)

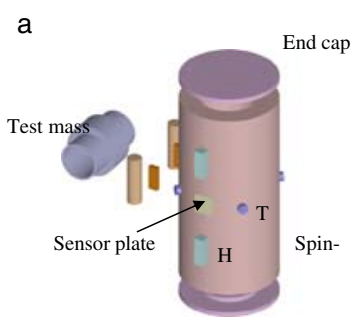

b

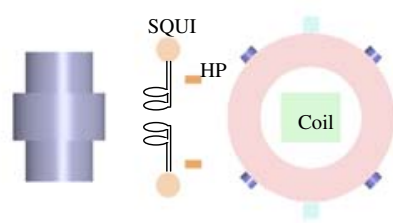

test mass pairs in MEPE. The entire IRSC payload is located within the liquid helium dewar and maintained at $1.8 \mathrm{~K}$. A set of magnetic and thermal sensors are incorporated to diagnose potential systematic effects (described below). These comprise SQUIDs, in both magnetometer and gradiometer configurations, together with a Hall probe (HP) to search for modulated and static magnetic fields, and heaters $(\mathrm{H})$ and thermometers $(\mathrm{T})$ to mimic and measure any heating effects. The spin-source, located approximately $12 \mathrm{~cm}$ from the test mass, comprises a cylinder of high permeability ferromagnetic material with a high saturation field at low temperature. Figure 7 shows, on the left, a schematic of the spin source with inner/outer dimensions of $3 / 5 \mathrm{~cm}$ and a length of $20 \mathrm{~cm}$. The spin-source generates a macroscopic alignment of spins but at the same time minimises magnetic field leakage to avoid systematic effects through interactions with impurities in the test mass. This is done using a high permeability magnetic shield. A coil on the inside of shield polarises the spins in the shield. This novel design [18] ensures that the field generated by the coil is trapped in the high permeability material. To fully suppress magnetic field leakage the source has high permeability caps which totally enclose the coil. In Fig. 7 a vector field plot of the contributions from the coil and the shield show that outside the spin source the residual magnetic field is negligible. To further suppress any leakage there is a superconducting film of niobium sputter coated onto the outside of the spin-source. This does not affect the spin-coupling signal as superconductivity arises from paired electrons (Cooper pairs) with zero net spin. A convenient feature is that the spins can be modulated electronically, by reversing the current in the coil. The experimental search will look for a spin coupling torque generated at a specific known frequency. To maximise the signal, the spin-source will be operated close to the saturation field of Mu-Metal of approximately $0.7 \mathrm{~T}$. The coil, which polarises the spin-source, must be fabricated from $0.25 \mathrm{~mm}$

Fig. 7 a Schematic of the spin source showing the polarising coil, the high permeability cylinder and the superconducting coat. b Vector magnetic field plot of the contributions from the coil and the high permeability material

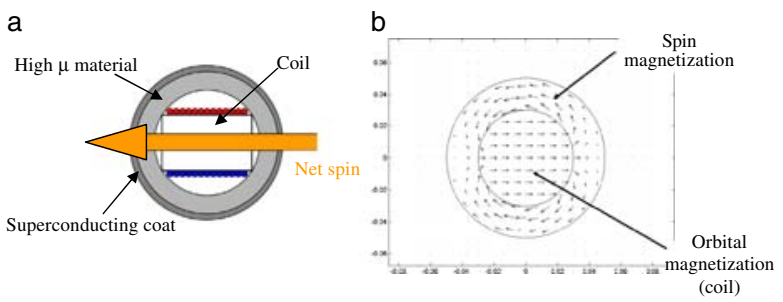


diameter niobium-tin $(\mathrm{Nb}-\mathrm{Sn})$ wire with a superconducting transition at $23 \mathrm{~K}$ and a critical field of $25 \mathrm{~T}$ at $4.2 \mathrm{~K}$. A coil with 4,000 turns and carrying $10 \mathrm{~A}$ achieves the required magnetic field. The current has to be periodically stored and quenched to modulate the spin-coupling signal every 1,000 s.

The main problems are systematic effects mimicing spin-coupling interaction. Typically these effects are either magnetic or thermal. Magnetic effects arise from coupling between leakage field/gradient and magnetic impurities in the test mass, and can appear at both the modulation frequency and its first harmonic ( $f$ and $2 f$ ). SQUIDs and Hall probes in between the spinsource and the test mass (see Fig. 6) will measure the residual leakage field and place limits on magnetic impurities in the test mass. The Hall probe provides information on the static leakage field due to stable trapped flux in the superconducting films and the residual field in the spacecraft environment. SQUIDs, in magnetometer and gradiometer configurations, provide information on modulated components of the leakage magnetic field due to trapped flux moving in response to the applied field. Systemic magnetic effects are only a problem if there is a net magnetic moment on the test mass due, for example, to ferromagnetic impurities. These can be looked for by modulating the test mass along the sensitive axis (using the test mass suspension) and searching for magnetic fields coupled into the SQUID. Thermal systematics, which can result from hysteretic or eddy current heating in the spin source, are typically $2 f$ contributions and appear out of the measurement band, unless the modulation current has a DC offset. Care needs to be taken to limit DC offsets to $<1 \mathrm{~mA}$. Magnetostriction can be a problem if the spin source has non-uniform coefficients of expansion. Energising the spin source with opposite current polarities can shift the centre of mass of the spin source at the modulation frequency, giving a gravitational coupling to the test mass at the signal frequency. This is not expected to be a major systematic uncertainty and it will be possible to measure the spin-source deformation either with an interferometer or capacitive sensor. Figure 6 shows the spin source with plates attached to either side. These plates form a capacitive sensor which can monitor the spin-source and infer whether the expansion due to magnetostriction is uniform for both polarities of current. The required position sensitivity to limit these systematic effects is $\sim 0.1 \mathrm{~nm}$. Heaters and thermometers, positioned close to the spin-source, measure and mimic the heating effects for systematic shakedown tests. Each experiment will require approximately $10^{4} \mathrm{~s}$. In addition to the systematic tests a spin-coupling measurement will be performed at a period of $1,000 \mathrm{~s}$ and will run for up to $10^{6} \mathrm{~s}$. The systematic tests will be repeated at the end of the run in order to assess the stability of the trapped flux and net polarisation on the test mass. The total experiment time is likely to be $10-15$ days. An acceleration sensitivity of $10^{-14} \mathrm{~ms}^{-2} / \sqrt{\mathrm{Hz}}$ at a frequency of $1 \mathrm{mHz}$, together with an integration time of $10^{6} \mathrm{~s}$, would result in the limits shown in the right-hand panel in Fig. 8. At ranges of $10 \mathrm{~cm}$ this improves on current constraints from ground based experiments by up to 3 orders of magnitude. A short-range spin-coupling test is also possible in principle using atom interferometry. Placing spin-sources close the matter wave paths 

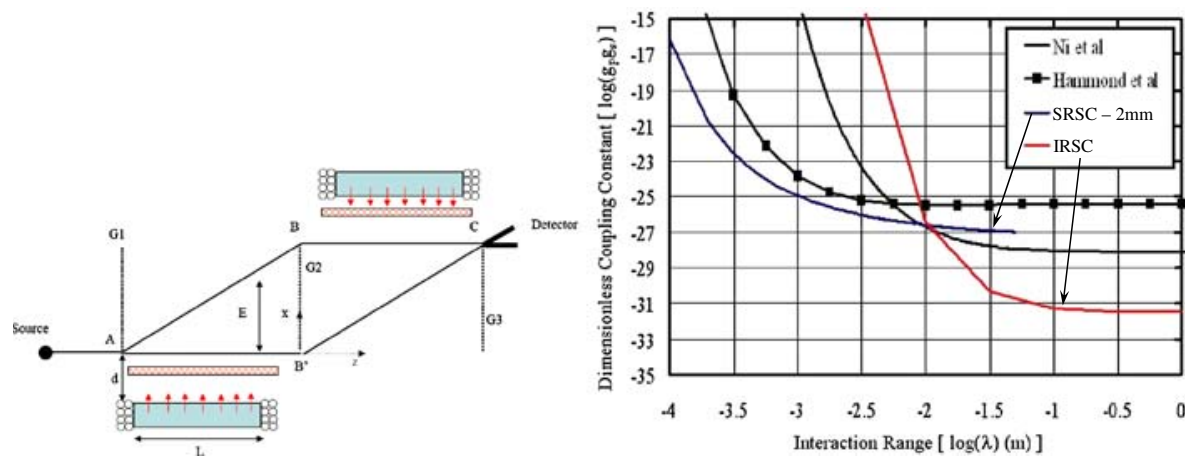

Fig. 8 Left a short-range spin-coupling test using atom interferometry. Right sensitivity for both IRSC, for an integration time of $10^{6} \mathrm{~s}$ and SRSC with single-shot atom interferometry using a simplistic analysis

in a spatially dispersed arrangement, as shown in Fig. 8, gives an additional phase shift of $\delta \phi=\frac{1}{\hbar} \int \frac{d r}{v} V(r)$, with $V(r)=g_{p} g_{s} \frac{\hbar^{2}}{8 \pi m_{\text {spin }}} \sigma \cdot r\left[\frac{1}{\lambda r}+\frac{1}{r^{2}}\right] \exp (-r / \lambda)$. Assuming $10^{8}$ caesium atoms traveling at $1 \mathrm{~m} / \mathrm{s}$ with an effective temperature $\sim 1 \mu \mathrm{K}$, and a spin source of dimensions $5 \mathrm{~mm} \times 0.25 \mathrm{~m}$ polarised with $50 \mathrm{~A}$ (100 turns). Figure 8 (right) shows the sensitivity (green curve) of an idealised single-shot measurement using atom interferometry compared with current ground-based results. In principle the sensitivity should improve as $\sqrt{N}$ where $N$ is the number of repeat shots which could be as high as $10^{6}$. However a major limitation with this technique is the beam divergence which is much larger than the 75-375 $\mu \mathrm{m}$ separations assumed in the simplistic calculations so far. This option is worthy further study but is not yet on firm enough ground to adopt as baseline. One potential improvement might be to use a guided atom laser as the source as already noted earlier.

\subsection{Macroscopic equivalence principle experiment}

The macroscopic test of the equivalence principle will be carried out using a free-fall test in Earth orbit as shown in Fig. 9. Four test mass pairs will be used, each constituting a differential accelerometer. The test mass materials have been chosen to provide sensitivity to the dilaton inspired violations of Damour and Polyakov [1]. Three of the pairs (1-3) provide a closure relationship giving a vital systematic check for any positive violation discovered. The fourth mass pair (4) is orientated such that it should not see any signal. It is otherwise identical to mass pair (1) and so again provides a systematic check. It also acts as a sensitive measurement mass for the intermediate ISL experiment. The masses are all operated at LHe temperature within the cryogenic science module. Readout will be a combination of magnetic (SQUID), optical and capacitative. The measurement uses the Earth as a source mass, but in order to move the signal away from once per orbit, which will be contaminated by 

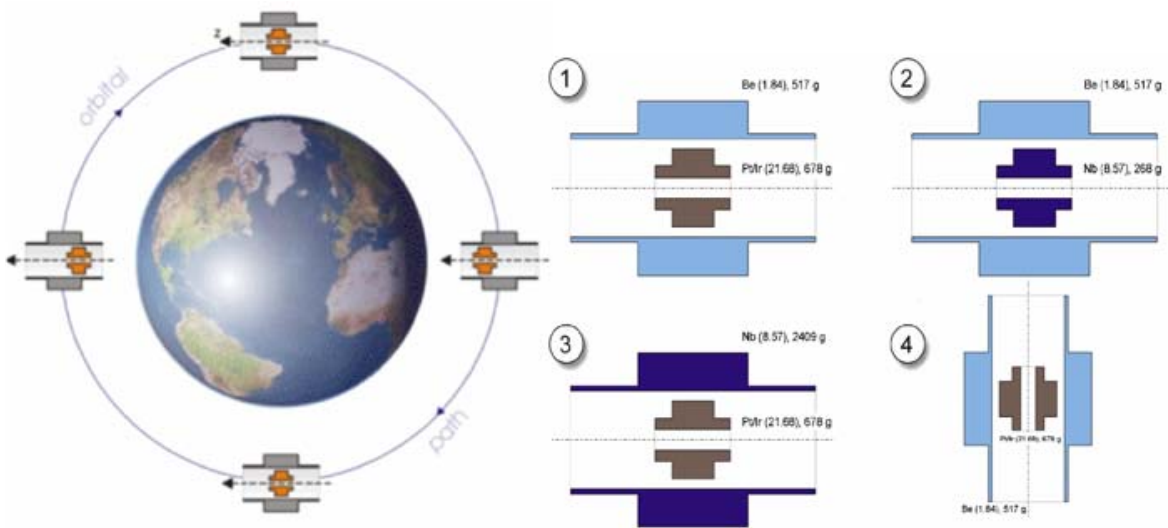

Fig. 9 Orbital free-fall equivalence principle test

other non-ideal effects, the payload will be spun about the probe axis at about three revolutions per orbit.

An orbital free-fall experiment of this type has already been studied in excruciating detail within ESA/NASA by the STEP project [55]. This proposal, although distinct from STEP, retains all the key features relevant to the noise and performance budget. An example noise budget from STEP is shown in Fig. 10. The figures are calculated for a single 20 orbit observation. In the example shown the dominant contributions are from read-out noise and electric potential variations. The science goal of GAUGE is to achieve a sensitivity of 1 part in $10^{18}$. We will repeat the experiment at least 20 times with planned experimental set-ups designed to explore systematics and give robustness to the result.
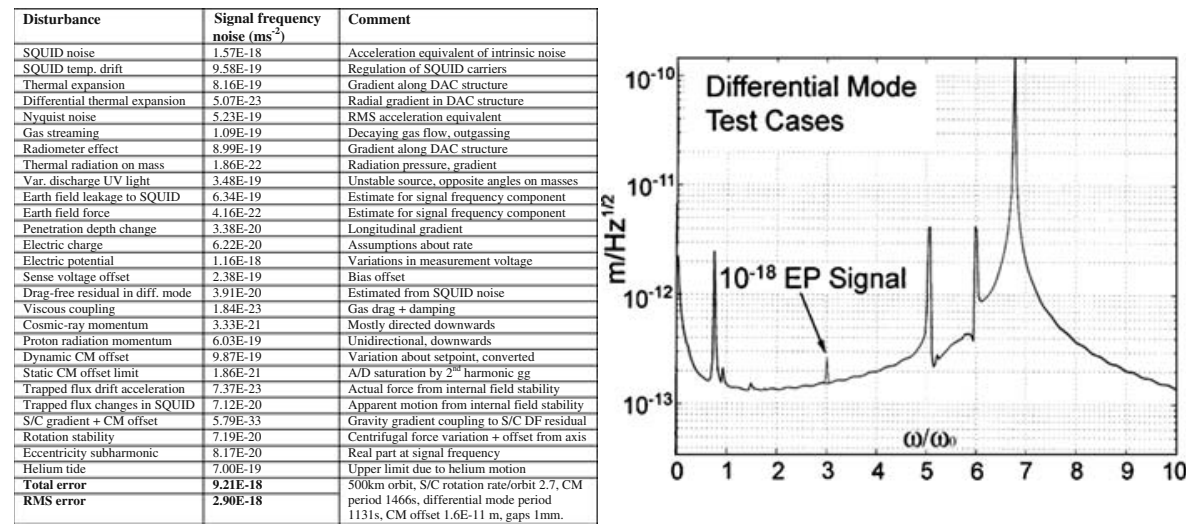

Fig. 10 Error budget and drag-free simulation results for STEP 


\section{Basic spacecraft key factors}

The principle behind the GAUGE spacecraft design has been to identify, as far as possible, existing concepts and technologies that meet the individual instrument requirements. In particular we identify the LISA Pathfinder Science Module (LPSM) including its Inertial Sensor and DFACS. Its functions for GAUGE will be:

- Provision of standard services (Power, Telemetry, etc.) to all payload instruments

- Physical accommodation of all payload instrument control electronics

- Provision of a drag-free environment, either stand-alone using the LTP or in conjunction with sensors within the other experiments

- Physical accommodation of GAUGE Atom Interferometer

- Use of the LTP test masses as part of an inverse square law (ISL) experiment

Attached to the LPSM is the Cryogenic Science Module (CSM), based on existing designs for open-cycle LHe dewars. Its main functions for GAUGE will be:

- Provision of cryogenic environment

- Accommodation of the macroscopic equivalence principle proof-masses

- Accommodation of the spin source for the spin-coupling experiment

- Accommodation of a dedicated test mass pair for the ISL experiment

- Provision of drag-free components, either stand-alone or in conjunction with the LTP, including provision of cold gas for possible thrust control

LISA Pathfinder Science Module Design for GAUGE The science module benefits from the heritage of LISA Pathfinder, due to launch in 2009. LPSM consists of an octagonal structure with a fixed solar array mounted to the upper panel. Volume for the room temperature payload including the LTP and the Atom Interferometer is provided within the central cylinder. In addition the LPSM accommodates all service module equipments required for the mission. Wherever possible LISA Pathfinder systems have been baselined. The electrical system and AOCS equipments are identical to those of LPF. The structure only differs in that the lower interface from the central cylinder is to the CSM rather than a propulsion module.

Cryogenic Science Module for GAUGE Some experiments on GAUGE benefit from operating elements in the CSM in conjunction with elements in LPSM. A key requirement, is that vibrational noise be kept to an absolute minimum. This, together with the need for LEO, precludes the use of active cryo-coolers based on Stirling compressors and mechanical Joule-Thompson systems (too noisy) or cryo-coolers based on sorption coolers and radiative cooling (insufficient performance in LEO). Consequently, the design is based on a passive LHe dewar, sized to ensure sufficient life-time. This has the benefit that He boil-off gas can be used by the drag-free system, as successfully 
demonstrated by GP-B. There is a trade-off between capacity and life-time on the one hand, and mass on the other. An additional constraint is set by available launch fairing volume (VEGA). The most relevant dewar heritage comes from GP-B and STEP [56]. GP-B employed a 25001 dewar and had a lifetime of approximately 14 months, whereas the baseline STEP dewar has 2301 and is designed for total lifetime (including margin) of approximately 8 months. For GAUGE, different experiments relying on cryogenic conditions will have to be operated in sequence, and as a result the overall life-time needs to be the sum of the anticipated operating time for these experiments. As a first approach, it has been assumed that the outer diameter of a STEP-like dewar can be increased to $1.5 \mathrm{~m}$ and still remain shadowed by the solar array at all times. No attempt has been made to assess secondary effects, such as additional residual atmospheric drag or mechanical strengthening or stiffening of the dewar. With a $1.5 \mathrm{~m}$ diameter the LHe capacity increases to approximately 5001 . Assuming a heat load of $25 \mathrm{~mW}$ (as for STEP) this provides a life-time compatible with the science requirements, including margin, of approximately 20 months.

\section{References}

1. Damour, T., Polyakov, A.M.: The string dilaton and a least coupling principle. Nucl. Phys. B 423, 532-558 (1994)

2. Damour, T., Federico, P., Gabriele, V.: Runaway dilaton and equivalence principle violations. Phys. Rev. Lett. 89, 081601 (2002)

3. Sandvik, H.B., Barrow, J.D., Magueijo, J.: A simple cosmology with a varying fine structure constant. Phys. Rev. Letts. 88, 031302 (2002)

4. Fischbach, E., Kraus, D.E., Talmadge, C., Tadic, D.: Higher order weak interactions and the equivalence principle. Phys. Rev. D 52, 5417-5427 (1995)

5. Peccei, R.D., Quinn, H.R.: CP conservation in the presence of pseudoparticles. Phys. Rev. Lett. 38, 1440-1443 (1977)

6. Fayet, P.: New interactions and the standard models. Class. Quantum Grav. 13, A19-A33 (1996)

7. Anselm, A.A., Uraltsev, N.G.: A second massless axion? Phys. Lett. B 114, 39-41 (1982)

8. Wilcezk, F.: Axions and family symmetry breaking. Phys. Rev. Lett. 49, 1549-1552 (1982)

9. Bardeen, W.A., Leung, C.N., Love, S.T.: Dilaton and chiral-symmetry breaking. Phys. Rev. Lett. 56, 1230-1233 (1986)

10. Moody, J.E., Wilczek, F.: New macroscopic forces? Phys. Rev. D 30, 130-138 (1984)

11. Raffelt, G.G.: Axions - motivation, limits and searches. arXiv:hep-ph/0611118v1 (2006)

12. Asztalos, S.J., et al.: Improved rf cavity search for halo axions. Phys. Rev. D. 69, 011101 (2004)

13. Zioutas, K., et al.: First results from the CERN axion solar telescope. Phys. Rev. Lett. 94, $121301(2005)$

14. Zavattini, E., et al.: Experimental observation of optical rotation generated in vacuum by a magnetic field. Phys. Rev. Lett. 96, 110406 (2006)

15. Heckel, B., et al.: New CP-violation and preferred-frame tests with polarized electrons. Phys. Rev. Lett. 97, 021603 (2006)

16. Youdin, A.N., et al.: Limits on spin-mass couplings within the axion window. Phys. Rev. Lett. 77, 2170-2173 (1996)

17. Ni, W.-T., et al.: Search for an axion-like spin coupling using a paramagnetic salt with a dc SQUID. Phys. Rev. Lett. 82, 2439-2442 (1999)

18. Hammond, G., et al.: New constraints on short-range forces coupling mass to intrinsic spin. Phys. Rev. Lett. 98, 081101 (2007) 
19. Jaekel, M.-T., Reynaud, S.: Gravitational quantum limit for length measurements. Phys. Lett. A 185, 143-148 (1994)

20. Klinkhamer, F.R.: Fundamental length scale of quantum spacetime foam. JETP Lett. 86, 73 (2007)

21. Amelino-Camelia, G.: Gravity-wave interferometers as probes of a low-energy effective quantum gravity. Phys. Rev. D 62, 024015 (2000)

22. Schiller, S., et al.: Experimental limits for low-frequency space-time fluctuations from ultrastable optical resonators. Phys. Rev. D 69, 027504 (2004)

23. Ellis, J., et al.: Space-time foam may violate the principle of equivalence. Int. J. Mod. Phys. A 19, 4413-4430 (2004)

24. Bernadotte, S., Klinkhamer, F.R.: Bounds on length scales of classical spacetime foam models. Phys. Rev. D 75, 024028 (2007)

25. Amelino-Camelia, G., et al.: Tests of quantum gravity from observations of $\gamma$-ray bursts. Nature 393, 763-765 (1998)

26. Alfaro, J., Morales-Tecotl, H.A., Urrutia, L.F.: Quantum gravity corrections to neutrino propagation. Phys. Rev. Lett. 84, 2318-2321 (2000)

27. Camacho, A.: Generalized uncertainty principle and deformed dispersion relation induced by nonconformal metric fluctuations. Gen. Rel. Grav. 34, 1839-1845 (2002)

28. Hu, B.L., Verdaguer, E.: Stochastic gravity: theory and applications. Living Rev. Relativity 7 , 3 (2004)

29. Ford, L.H.: Stochastic spacetime and Brownian motion of test particles. Int. J. Theor. Phys. 44, 1753-1768 (2005)

30. Camacho, A.: Decoherence and bare mass induced by nonconformal metric fluctuations. Gen. Rel. Grav. 35, 319-325 (2003)

31. Weinberg, S.: The cosmological constant problem. Rev. Mod. Phys. 61, 1-23 (1989)

32. Harris, B.W., Chen, F., Mohideen, U.: Precision measurement of the casimir force using gold surfaces. Phys. Rev. A 62, 052109 (2000)

33. Welton, T.A.: Some observable effects of the quantum-mechanical fluctuations of the electromagnetic field. Phys. Rev. 74, 1157-1167 (1948)

34. Senitzky, I.R.: Incoherence, quantum fluctuations, and noise. Phys. Rev. 128, 2864-2870 (1962)

35. Koch, R.H., van Harlingen, D., Clarke, J.: Measurements of quantum noise in resistively shunted Josephson junctions. Phys. Rev. B 26, 74 (1982)

36. Ford, L.H.: Quantum vacuum energy in general relativity. Phys. Rev. D 11, 3370 (1975)

37. Saunders, S.: In: Kuhlmann, M., Lyre, H., Wayne, A. (eds.) Onthological Aspects of Quantum Field Theory. World Scientific, Singapore (2002)

38. Carroll, S.M.: The cosmological constant. Living Rev. Rel. 4, 1 (2001)

39. Amelino-Camelia, G., Kowalski-Glikman, J. (Eds.): Planck Scale Effects in Astrophysics and Cosmology. Springer, Berlin (2005)

40. Wald, R.M.: Quantum Field Theory in Curved Spacetime and Black Hole Thermodynamics. University of Chicago Press, London (1994)

41. Hawking, S.W.: Black hole explosions? Nature 248, 30 (1974)

42. Page, D.N., Geilker, C.D.: Indirect evidence for quantum gravity. Phys. Rev. Lett. 47, 979 (1981)

43. Van den Bergh, N., et al: Conformally invariant fields in conformally flat spacetime. Class. Quantum Grav. 7, 2267 (1990)

44. Unruh, W.G., Zurek, W.H.: Reduction of a wave packet in quantum brownian motion. Phys. Rev. D 40, 1071 (1989)

45. Breuer, H.-P., Petruccione, F.: Destruction of quantum coherence through emission of bremsstrahlung. Phys. Rev. A 63, 032102 (2001)

46. Ford, L.H.: Electromagnetic vacuum fluctuations and electron coherence. Phys. Rev. D 47, 5571 (1993)

47. Sonnentag, P., Hasselbach, F.: Measurement of decoherence of electron waves and visualization of the quantum-classical transition. Phys. Rev. Lett. 98, 200402 (2007)

48. Penrose, R.: Quantum computation, entanglement and state reduction. Phil. Trans. R. Soc. Lond. A 356, 1927 (1998)

49. Diosi, L.: Notes on certain newton gravity mechanisms of wavefunction localization and decoherence. J. Phys. A 40, 2989 (2007)

50. Lamine, B., Herve, R., Lambrecht, A., Reynaud, S.: Ultimate decoherence border for matterwave interferometry. Phys. Rev. Lett. 96, 050405 (2006) 
51. Wang, C.H.-T., Bingham, R., Mendonca, J.T.: Quantum gravitational decoherence of matter waves. Class. Quantum Grav. 23, L59 (2006)

52. Turyshev, S.G., et al.: Space-based research in fundamental physics and quantum technologies. Int. J. Mod. Phys. D 16, 1879 (2007)

53. Dolesi, R., et al.: Gravitational sensor for LISA and its technology demonstration mission. Class. Quant. Grav. 20, S99 (2003)

54. Guerin, W., Riou, J.-F., Gaebler, J.P., Josse, V., Bouyer, P., Aspect, A.: Guided quasicontinuous atom laser. Phys. Rev. Lett. 97(20), 200402 (2006)

55. Sumner, T.J., et al.: STEP (Satellite Test of the Equivalence Principle). Adv. Space Res. 39, 254 (2007)

56. Mester, J., et al.: Gravitational experiments in space: gravity probe B and STEP. Nucl. Phys. B Proc. Suppl. 134, 147 (2004) 